\title{
Editorial
}

\section{Transport Phenomena in Porous Media and Fractal Geometry}

\author{
Peng Xu, ${ }^{1}$ Jianchao Cai, ${ }^{2}$ Agus Pulung Sasmito, ${ }^{3}$ Sachin Vinayak Jangam, ${ }^{4}$ and Boming Yu $^{5}$ \\ ${ }^{1}$ College of Science, China Jiliang University, Hangzhou 310018, China \\ ${ }^{2}$ Hubei Subsurface Multi-Scale Imaging Key Laboratory, Institute of Geophysics and Geomatics, \\ China University of Geosciences, Wuhan 430074, China \\ ${ }^{3}$ Department of Mining and Materials Engineering, McGill University, Montreal, QC, Canada H3A 2A7 \\ ${ }^{4}$ Department of Chemical and Biomolecular Engineering, National University of Singapore, Singapore 117585 \\ ${ }^{5}$ School of Physics, Huazhong University of Science and Technology, Wuhan 430074, China \\ Correspondence should be addressed to Peng Xu; pengxuhust@gmail.com
}

Received 1 July 2015; Accepted 2 July 2015

Copyright (C) 2015 Peng Xu et al. This is an open access article distributed under the Creative Commons Attribution License, which permits unrestricted use, distribution, and reproduction in any medium, provided the original work is properly cited.

The concept of porous media is used in many areas of applied science and engineering [1-3]. Transport phenomena in porous media, including single phase and multiphase fluid flow through porous media, heat transfer in porous media, and electrical and acoustical transport in porous media, are a subject of most common interest and have emerged as a separate field of study [4-6]. Transport phenomena in porous media, from microscopic to upward scales, cover general theories behind flow and transport in porous media and form the basis for deterministic and stochastic models that describe them. However, the microstructures and transport mechanisms in porous media are very complex in nature. Fractal geometry has been shown to have potential in the analysis of flow and transport properties of porous media (consult recent special issue [7-11]). This special issue includes a number of recent works on transport phenomenon and applications of fractal geometry theory in porous media.

The first group of papers focuses on the transport phenomena in porous media. L. Wang et al. presented the applicability of different fluid media to measure effective stress coefficient for rock permeability. T. Huang et al. established a multiscale comprehensive mathematical model to simulate different flow regimes. C. Li et al. constructed a mathematical model to describe gas from porous coalbed methane reservoirs with complex boundary conditions. A water alternating $\mathrm{CO}_{2}$-LPG enhanced oil recovery simulation model was developed by J. Cho et al.

For transport process in complex reservoir media, J. Guo et al., D. Li et al., Z. Jia et al., and W. Xie et al. studied the transient flow in fractured/shale reservoirs. L. Yuan et al. proposed a model for local skin factor and productivity of horizontal well. L. You et al. investigated the adsorption stability of surfactant on the rock mineral surface. R. Cao et al. theoretically modeled the wettability variation during long-term water flooding. B. Zheng et al. reported the results of experimental investigation of flow resistance in a coal mine ventilation air methane preheated catalytic oxidation reactor. Y. Kang et al. measured the physical properties of samples from representative tight gas reservoirs before and after high temperature treatment. L. Zhang et al. developed a new compositional model to characterize non-Darcy flow in tight/shale gas reservoirs. L. Zhang et al. established a discrete network model for fracture-cave reservoirs to study fluid flow characteristics and pressure distributions.

Z. Dou et al. numerically simulated the immiscible liquid transport in cavity-fractures by Lattice Boltzmann (LB) method. Y. Xu et al. presented a LB model of the uniform velocity and driven convective thermal conductivity in a porous cavity. $\mathrm{H}$. $\mathrm{Lu}$ et al. investigated the hydraulic conductivity of soil under different wetting-drying cycles.

The second group of papers focuses on the applications of fractal geometry in porous media. M. A. Martín et al. provided an explanation to the complexity of spatial variability of chemicals or pollutants in soil via an extremely simple model. Z. Wang et al. presented a review on the major development on fractal analysis of porous metal materials. J. Zhao et al. used similar construction method of solution to solve mathematical models of spherical flow in a fractal reservoir. 
We hope that this special issue will help to further advance this multidisciplinary endeavor of fractal geometry theory and porous media.

\section{Acknowledgments}

The guest editors are thankful to all authors for their excellent contributions and to the anonymous referees for their efforts in providing valuable comments.

$$
\begin{array}{r}
\text { Peng Xu } \\
\text { Jianchao Cai } \\
\text { Agus Pulung Sasmito } \\
\text { Sachin Vinayak Jangam } \\
\text { Boming Yu }
\end{array}
$$

\section{References}

[1] M. Sahimi, "Flow phenomena in rocks: from continuum models to fractals, percolation, cellular automata, and simulated annealing," Reviews of Modern Physics, vol. 65, no. 4, pp. 1393$1534,1993$.

[2] B. M. Yu, "Analysis of flow in fractal porous media," Applied Mechanics Reviews, vol. 61, no. 5, Article ID 050801, 2008.

[3] P. Xu and B. Yu, "Developing a new form of permeability and Kozeny-Carman constant for homogeneous porous media by means of fractal geometry," Advances in Water Resources, vol. 31, no. 1, pp. 74-81, 2008.

[4] J. Cai, B. Yu, M. Zou, and L. Luo, "Fractal characterization of spontaneous co-current imbibition in porous media," Energy and Fuels, vol. 24, no. 3, pp. 1860-1867, 2010.

[5] A. Mirzaei-Paiaman and M. Masihi, "Scaling of recovery by cocurrent spontaneous imbibition in fractured petroleum reservoirs," Energy Technology, vol. 2, no. 2, pp. 166-175, 2014.

[6] M. Xu and H. Dehghanpour, "Advances in understanding wettability of gas shales," Energy Fuels, vol. 28, no. 7, pp. 43624375, 2014.

[7] E. Perfect, Y. Pachepsky, and M. A. Martin, "Fractal and multifractal models applied to porous media," Vadose Zone Journal, vol. 8, no. 1, pp. 174-176, 2009.

[8] J. Cai, F. San José Martiínez, M. A. Martín, and E. Perfect, “An introduction to modeling of flow and transport in fractal porous media: part I," Fractals, vol. 22, no. 3, Article ID 1402001, 2014.

[9] B. Ghanbarian, A. G. Hunt, R. P. Ewing, and T. E. Skinner, "Universal scaling of the formation factor in porous media derived by combining percolation and effective medium theories," Geophysical Research Letters, vol. 41, no. 11, pp. 3884-3890, 2014.

[10] J. Cai, F. San José Martínez, M. A. Martínez, and X. Hu, "An introduction to flow and transport in fractal models of porous media: part II," Fractals, vol. 23, no. 1, Article ID 1502001, 2015.

[11] J. Cai, L. Luo, R. Ye, X. Zeng, and X. Hu, "Recent advances on fractal modeling of permeability for fibrous porous media," Fractals, vol. 23, no. 1, Article ID 1540006, 2015. 

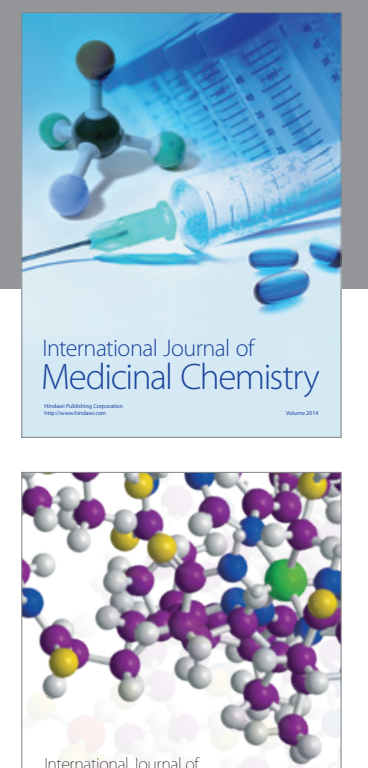

\section{Carbohydrate} Chemistry

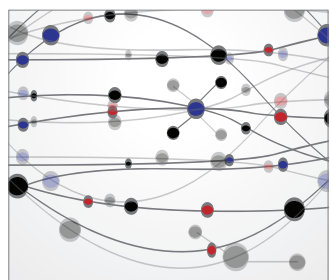

The Scientific World Journal
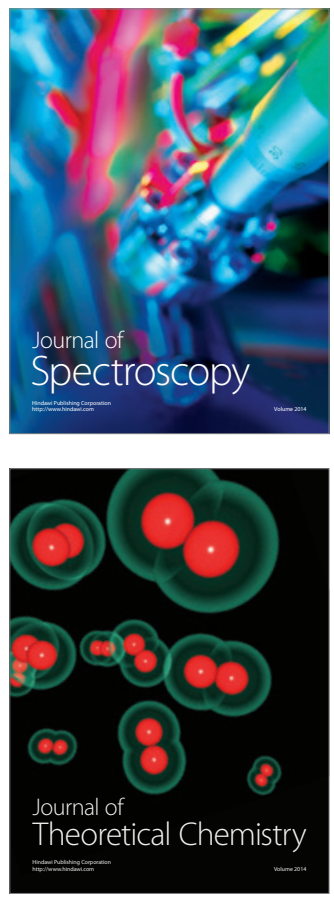
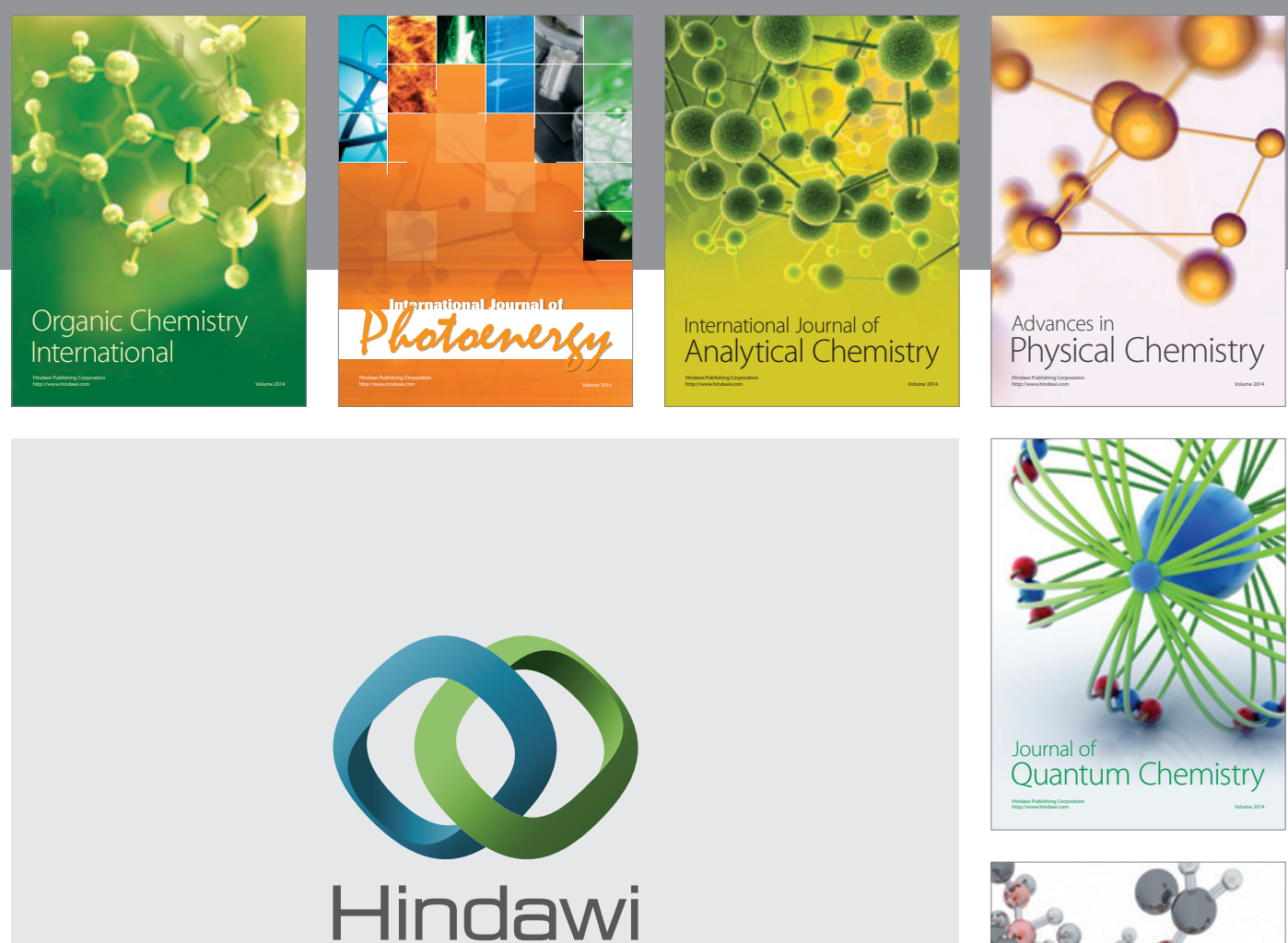

Submit your manuscripts at

http://www.hindawi.com

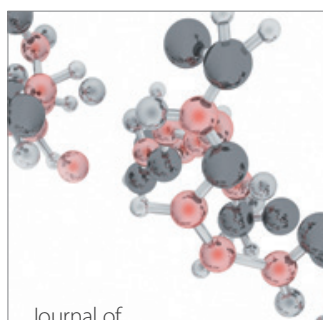

Analytical Methods

in Chemistry

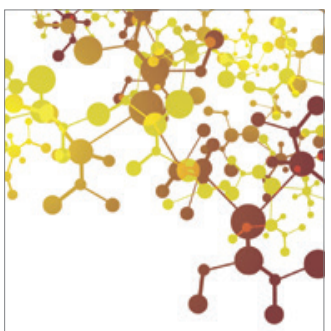

Journal of

Applied Chemistry

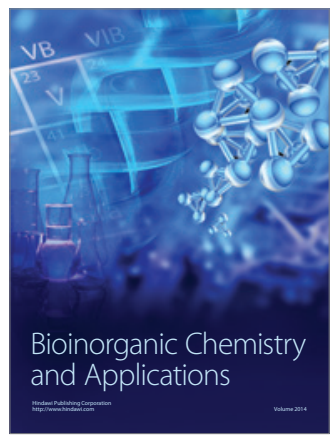

Inorganic Chemistry
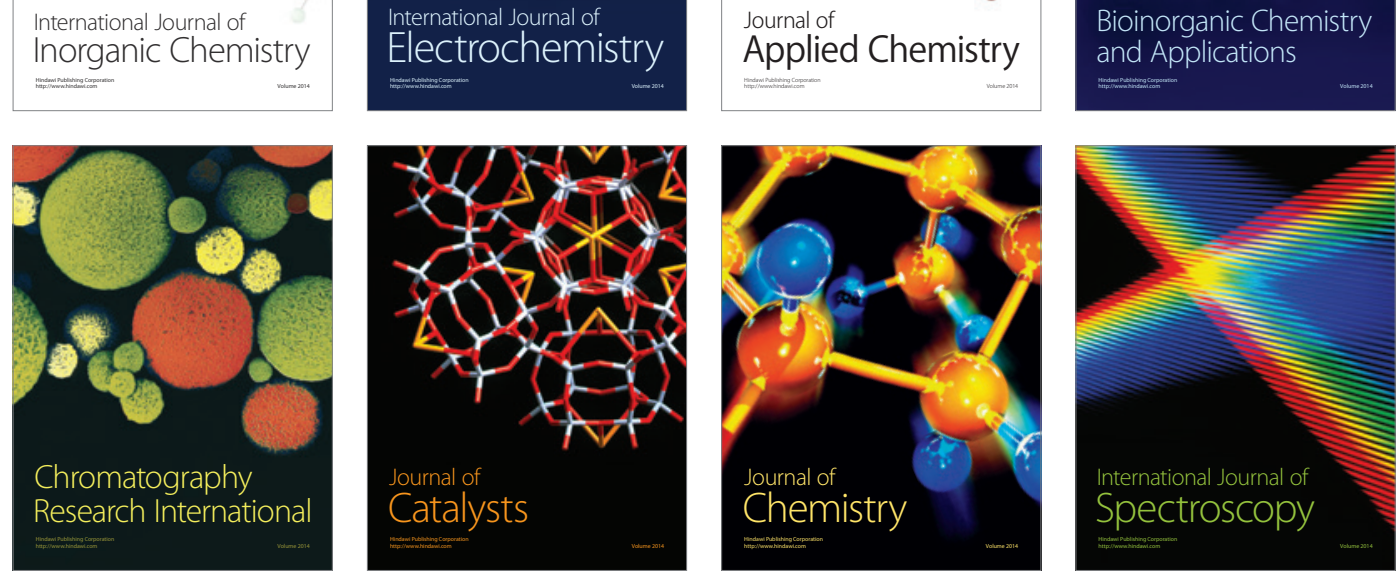